\title{
Foreword
}

\section{Overview of the Special Focus: New Paradigms for Biomedical Engineering and Biotechnology}

This Special Focus consists of selected papers that were presented at the 4th International Conference on Biomedical Engineering and Biotechnology (iCBEB2015). The meeting was held from August 18th-21st, 2015, in Shanghai, China, and information about the meeting can be perused online at http://2015.icbeb.org/. The meeting was the fourth in a yearly series of international conferences in which leading scientists from throughout the world have been invited to attend and to deliver talks on their work pertinent to the development of biomedical engineering techniques and technology. As was also typically the case in prior years, approximately 1,200 abstracts were received by the Program Committee, and approximately 200 of these were selected for oral presentation at the meeting. A number of others were selected for poster presentation. Of the 200 studies presented orally, 50 highly regarded works were determined to be of sufficient merit to be published in Technology and Health Care. These papers were first peer-reviewed, and suggestions were made for improvement, which was done prior to publishing.

I would like to thank Professor Feng Liu, PhD, of Wuhan University, Wuhan, China, who ran the meeting and who provided suggestions for best papers. Each year the meeting grows in stature and prestige, thanks to his guidance and to the assistance of his conference leadership team. Additionally, there is always a very nice cultural program accompanying the meeting, for the benefit of those who would like to enjoy and learn about the local surroundings and the environment for academic learning and research in the region. The iCBEB series becomes more widely know each year, with greater number of participants. Many attendees have commented on the helpfulness of the meeting for their understanding and education. The conference was attended by a mix of distinguished faculty, postdoctoral students, as well as graduate and undergraduate students. An important aim of the gathering was education - at all levels - on the latest cutting edge technology in the field of biomedical engineering and related areas. We believe that the reader will be interested in the selection of 50 articles published in this special focus. As they cover a wide range of topics, it is likely that some will be of special interest, and we think, helpfulness, for those working in the field.

Guest Editor

Edward J. Ciaccio, PhD

Division of Cardiology and Celiac Disease Center Department of Medicine P\&S 7-446

Columbia University

630 West 168th Street

New York, NY 10032, USA

E-mail: ciaccio@columbia.edu

0928-7329/16/\$35.00 @ 2016 - IOS Press and the authors. All rights reserved This article is published online with Open Access and distributed under the terms of the Creative Commons Attribution NonCommercial License. 